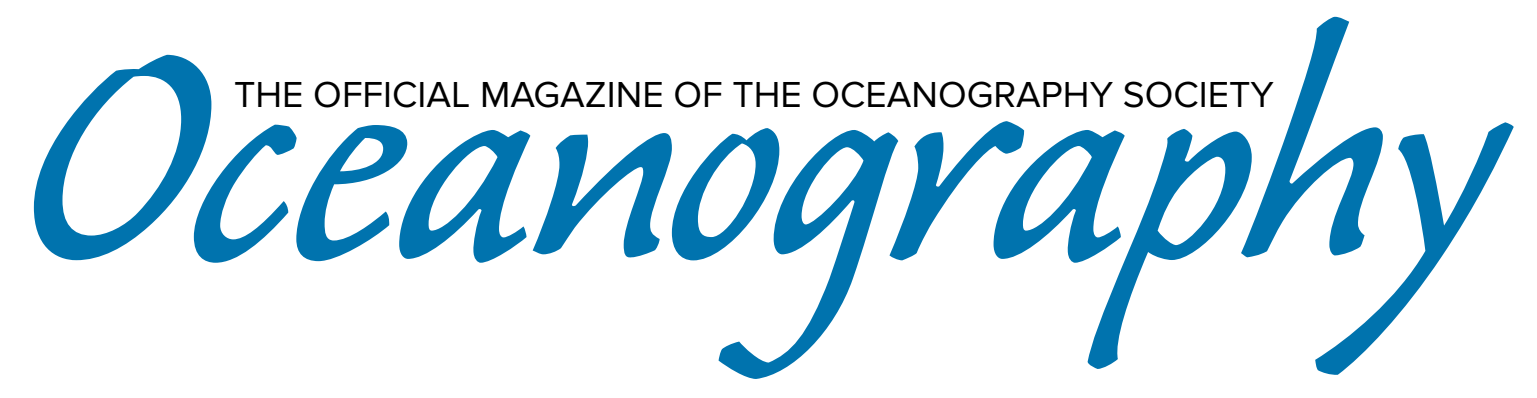

CITATION

Wheeler, M.H. 2018. Simplified models for equatorial waves with vertical structure.

Oceanography 31(3):36-41, https://doi.org/10.5670/oceanog.2018.307.

$\mathrm{DOI}$

https://doi.org/10.5670/oceanog.2018.307

COPYRIGHT

This article has been published in Oceanography, Volume 31, Number 3, a quarterly journal of The Oceanography Society. Copyright 2018 by The Oceanography Society. All rights reserved.

USAGE

Permission is granted to copy this article for use in teaching and research.

Republication, systematic reproduction, or collective redistribution of any portion of this article by photocopy machine, reposting, or other means is permitted only with the approval of The Oceanography Society. Send all correspondence to: info@tos.org or The Oceanography Society, 1 Research Court, Suite 450, Rockville, MD 20850, USA. 


\title{
Simplified Models for Equatorial Waves with Vertical Structure
}

\author{
By Miles H. Wheeler
}

\begin{abstract}
Explicitly solvable models have recently been developed for the interaction of waves with the Equatorial Undercurrent in an $f$-plane approximation. We observe that these analyses can be dramatically simplified by a Boussinesqtype approximation, and that the additional errors introduced are for the most part negligible. The approximate formulas are easier to interpret and show that critical layers occur if and only if the disturbances of the thermocline and free surface are in phase.
\end{abstract}

\section{INTRODUCTION}

One of the distinguishing features of the equatorial Pacific is the Equatorial Undercurrent (EUC), a strong eastward current that extends some $200 \mathrm{~m}$ below the surface and attains speeds of $1 \mathrm{~m} \mathrm{~s}^{-1}$ or more (Johnson et al., 2001). This is in contrast to the surface current, which is westward and driven by trade winds. Vertical stratification plays an important role, with a sharp pycnocline/thermocline at around $120 \mathrm{~m}$ depth. The simplest oceanographic models, based on so-called 1/1/2-layer shallow water equations, assume that there is no motion below the thermocline, and completely neglect the vertical structure of the EUC (McCreary, 1985). Nevertheless, they have been quite successful, for instance as components of larger models for El Niño and La Niña (Fedorov and Brown, 2009).

While detailed theories including the EUC are typically numerical (McPhaden et al., 1986), Constantin and Johnson (2015) introduced a linear model that can be solved explicitly. Focusing on the vertical structure of the EUC, they work in a nontraditional $f$-plane approximation rather than the usual $\beta$-plane and set all meridional velocities and variations to zero. This limits the validity of the theory to a band of at most $2^{\circ}$ latitude about the equator. By approximating the EUC as a purely zonal flow with piecewise-linear dependence on depth, they are able to obtain an algebraic relation between the speed $c$ and zonal wavenumber $k$ of a traveling wave. While explicit, this and other formulas in the model are quite complicated, and so the authors use various limiting procedures in different cases to produce more tractable formulas that are easier to interpret and compare with available data.

Subsequent mathematical research into related twodimensional models has centered on obtaining nonlinear Hamiltonian equations for the thermocline and free surface (Compelli and Ivanov, 2015; Constantin et al., 2016; Compelli and Ivanov, 2017; Ionescu-Kruse and Martin, 2017). These equations can be used to give Hamiltonian derivations of various nonlinear long-wave models such as the Korteweg-de Vries equation. See Craig et al. (2005) and the references cited therein for earlier results on irrotational flows, where only uniform currents are possible. For more on the modeling of zonal currents with flow reversal in absence of waves, see Constantin and Johnson (2016) as well as the article by Basu (2018) in this issue.

By including the vertical structure of the EUC, both Constantin and Johnson (2015) and McPhaden et al. (1986) allow for critical layers where the speed of the EUC coincides with the wave speed. In inviscid models, these open up into dramatic "Kelvin's cat's eye" patterns (see Figure 1). Critical layers are known to be important mechanisms for instability, and a full accounting of their properties requires the introduction of viscous and/or nonlinear effects (Maslowe, 1986).

In this note we consider a two-layer model along the lines of Constantin and Johnson (2015), but perform an expansion in the density variation across the thermocline. Such Boussinesqtype approximations are commonplace in oceanography, but do not appear to have been considered for this particular family of models. An immediate advantage is that the formulas for the wave speed and relative amplitudes of the free surface and thermocline are drastically simplified. Questions about critical layers, the validity of the rigid-lid approximation, and the effect of Coriolis forces can then be more easily understood. Unlike 
the limiting procedures employed by Constantin and Johnson (2015), the approximation does not involve additional assumptions on the wavelength, and indeed appears to be extremely accurate for wavelengths anywhere from $5 \mathrm{~m}$ to $100 \mathrm{~km}$. We do filter out fast-moving gravity waves concentrated near the surface, but it is not clear that such waves have significant interactions with the EUC in the first place.

\section{MODEL AND APPROXIMATION}

\section{A Constantin-Johnson Type Model}

We begin with the incompressible Euler equations in a nontraditional $f$-plane approximation at the equator that retains Coriolis terms associated with the meridional component of Earth's angular velocity $\Omega$. This allows us to restrict to purely two-dimensional motion with no meridional dependence and no meridional velocity, but limits the applicability of the model to a band of perhaps $2^{\circ}$ around the equator (Johnson et al., 2001; Constantin and Johnson, 2015). To model the thermocline, we assume that there are two layers of constant density: a lower layer with density $\rho$ and mean depth $h_{1}$ and an upper layer with density $\rho-\Delta \rho$ and mean depth $h_{2}$ (see Figure 2 ). The pressure and normal fluid velocity are continuous across the thermocline, but waves in this inviscid model will have a (small) jump in normal velocity. The upper boundary of the ocean is assumed to be a free surface held at constant pressure (and not a rigid lid), while the bottom of the lower layer is a flat and impermeable ocean floor. We take the typical values $h_{1}=120 \mathrm{~m}$ and $h_{1}+h_{2}=4 \mathrm{~km}$ (Fedorov and Brown, 2009).

We approximate the EUC by a piecewise linear zonal velocity profile, illustrated schematically in Figure 3 . At the surface there is a negative/westward velocity $u_{\text {surf }}$, at the thermocline there is a positive/eastward velocity $u_{\text {therm }}$, and at the bottom there is no motion. We take the typical values $u_{\text {surf }}=-.25 \mathrm{~m} \mathrm{~s}^{-1}$ and $u_{\text {therm }}=1 \mathrm{~m} \mathrm{~s}^{-1}$ (Johnson et al., 2001). The advantage of a piecewise linear model is that the meridional component of the vorticity is constant in each layer, $\gamma_{2}=\left(u_{\text {surf }}-u_{\text {therm }}\right) / h_{2}$ in the upper layer and $\gamma_{1}=u_{\text {therm }} / h_{1}$ in the lower layer. This in turn allows us to consider irrotational perturbations, greatly simplifying the analysis. By taking a more complicated velocity profile, one could of course make a much better approximation to the EUC; Constantin and Johnson (2015) for instance use five layers. However, the algebra becomes significantly more complicated with each additional layer, and so for simplicity we restrict ourselves here to the two-layer case.

\section{A Boussinesq-Type Approximation}

We simplify the above model by recognizing

$$
\frac{\Delta \rho}{\rho} \ll 1
$$

as a small parameter. Typical values range between $4 \times 10^{-3}$ and $6 \times 10^{-3}$ (Fedorov and Brown, 2009; Constantin and Johnson, 2015); we take $\Delta \rho / \rho=5 \times 10^{-3}$. Using $U=1 \mathrm{~m} \mathrm{~s}^{-1}$ as a velocity
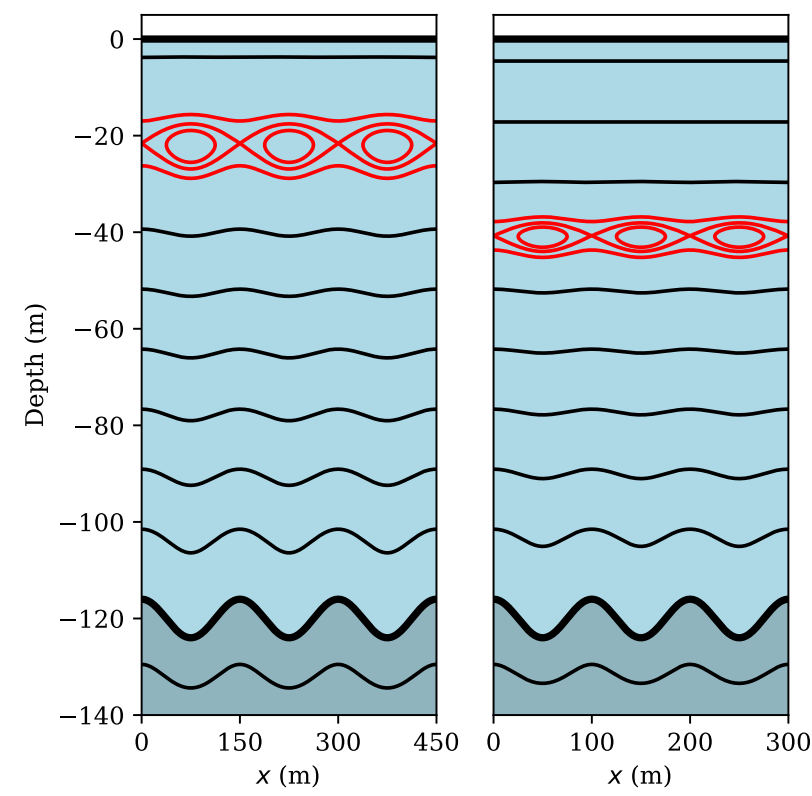

FIGURE 1. Streamlines for two waves with critical layers, shown in red. The thick black lines are the surface and the thermocline, the latter having an amplitude of $4 \mathrm{~m}$. The wave on the left travels at $c=-7 \mathrm{~cm} \mathrm{~s}^{-1}$, and the wave on the right at $c=9 \mathrm{~cm} \mathrm{~s}^{-1}$. Following the general pattern, the critical layer in the longer wave is closer to the surface.

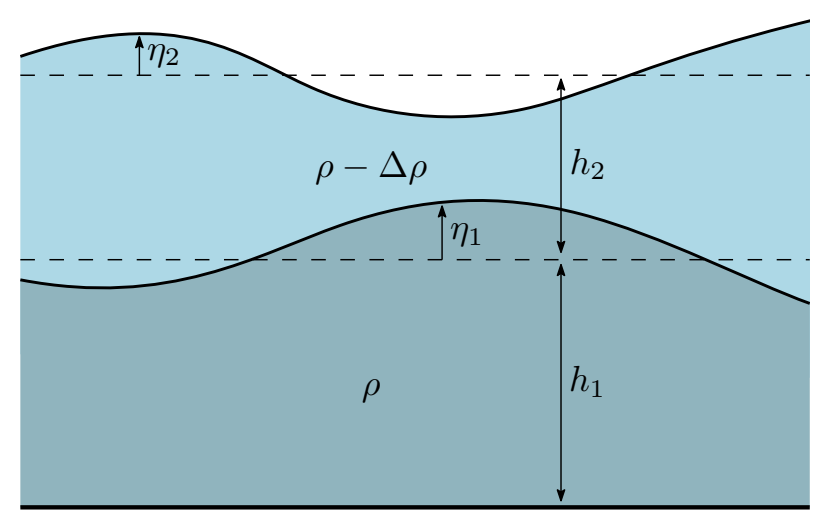

FIGURE 2. The thermocline is represented as a sharp interface between two regions of constant density, a lower layer with density $\rho$ and mean depth $h_{1}$, and an upper layer with density $\rho-\Delta \rho$ and mean depth $h_{2}$. The deviation of the thermocline is $\eta_{1}$ while the deviation of the free surface is $\eta_{2}$

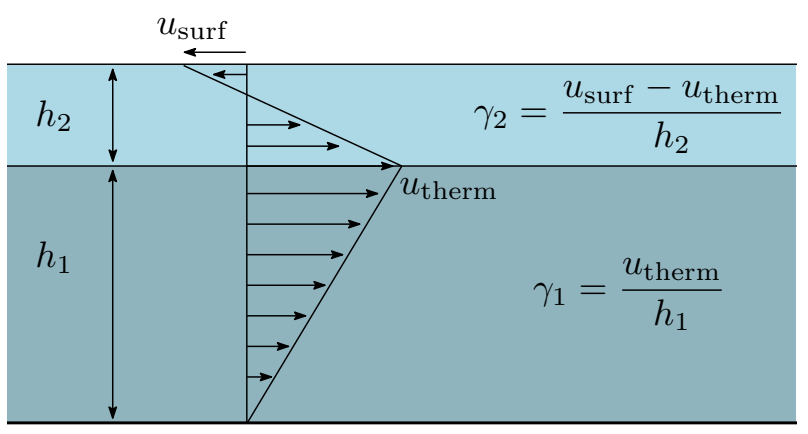

FIGURE 3. The Equatorial Undercurrent (EUC) is represented by a piecewise linear zonal velocity profile. The surface velocity $u_{\text {surf }}$ is westward due to trade winds, while at the thermocline there is an eastward current with strength $u_{\text {therm }}$, and at the bottom there is no motion. The (meridional) vorticities $\gamma_{1}$ and $\gamma_{2}$ in each layer are invariants of the motion. 


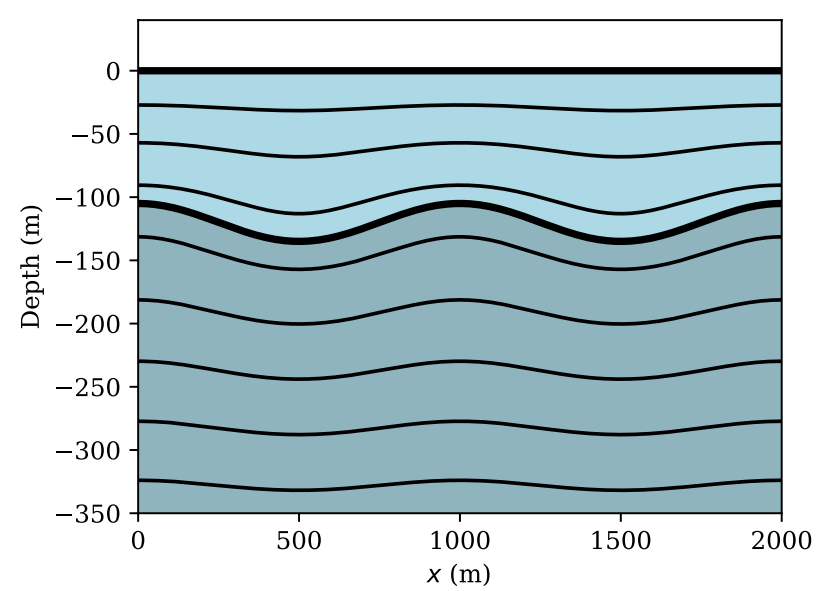

FIGURE 4. Streamlines for a wave calculated using the approximate model, with wavelength $1 \mathrm{~km}$, westward wave speed $c=2.25 \mathrm{~m} \mathrm{~s}^{-1}$, and amplitude $10 \mathrm{~m}$ at the thermocline. The thick lines are the free surface and the thermocline.

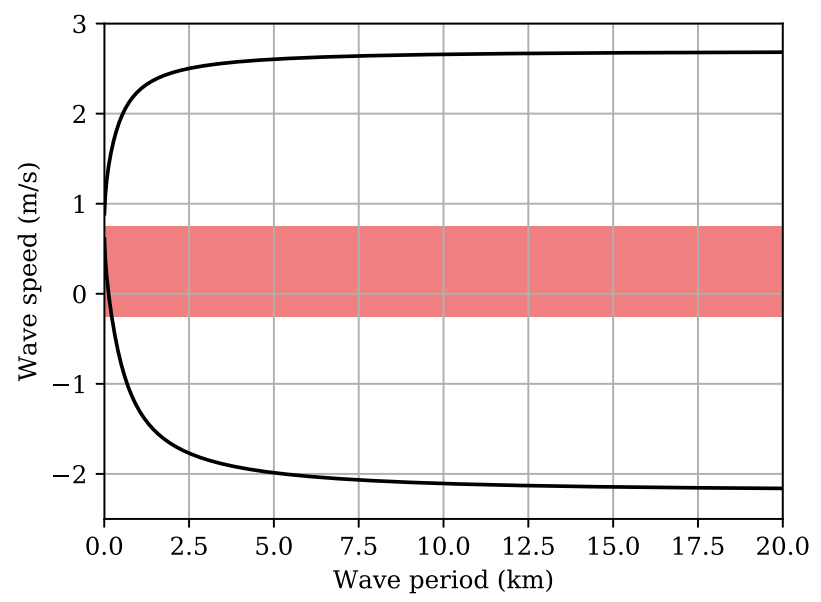

FIGURE 5. The wave speed $c$ versus wave period for the dispersion relation (1). Positive values of $c$ correspond to eastward propagating waves. The shortest waves travel at nearly the velocity $1 \mathrm{~m} \mathrm{~s}^{-1}$ of the thermocline. Points on the curve that lie in the red shaded region represent waves with critical layers.

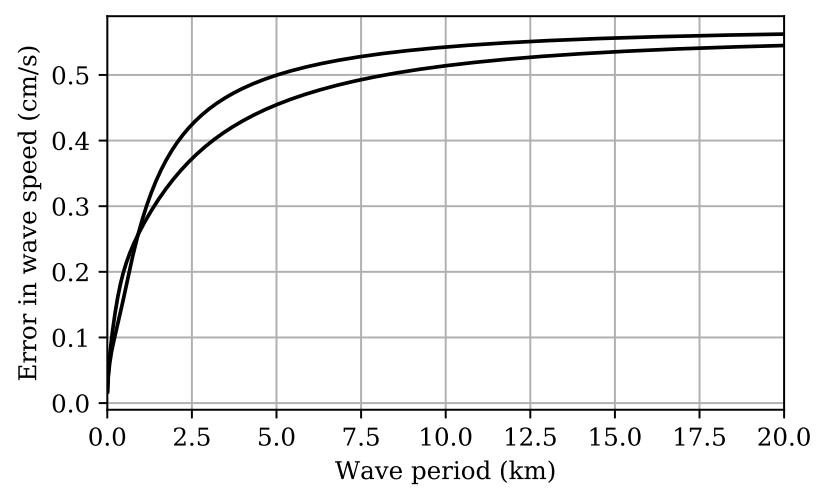

FIGURE 6. Absolute error between the wave speeds in Figure 5 and the corresponding wave speeds in the Constantin-Johnsontype model, as a function of the period. Note that the vertical scale is in $\mathrm{cm} \mathrm{s}^{-1}$; these represent relative errors of less than $0.3 \%$. scale for the EUC and $L=1 \mathrm{~km}$ a length scale, the Richardson number is then

$$
\mathrm{Ri}=\frac{g^{\prime} L}{U^{2}}=49,
$$

where $g^{\prime}=g \Delta \rho / \rho$ is the reduced gravity. The Richardson number is certainly not a small parameter here, and we will consider it fixed.

After using the above scales to nondimensionalize, we develop the unknowns as series in $\Delta \rho / \rho$ while holding the Richardson number Ri constant. In principle, this expansion can be carried out to any order, but here we will keep only the leading-order terms so that we arrive at linear equations.

\section{RESULTS}

The model described in the previous section and its approximation yield two sets of explicit formulas for the wave speed, velocity field, free surface, thermocline, and pressure in a wave with zonal wavenumber $k$. This allows us to, for instance, plot the streamlines of the $2 \mathrm{~km}$ wave in Figure 4 . In this section we discuss some of the more interesting formulas for the approximate model. Unfortunately, the corresponding formulas from the Constantin-Johnson type model are too long to reproduce here, but we do perform numerical comparisons for the parameter values from the previous section.

\section{Dispersion Relation}

The leading-order dispersion relation between the speed $c$ and zonal wavenumber $k$ in the Boussinesq approximation is

$$
\begin{aligned}
k\left(\operatorname{coth} k h_{1}\right. & \left.+\operatorname{coth} k h_{2}\right)\left(c-u_{\text {therm }}\right)^{2} \\
& +\left(\gamma_{1}-\gamma_{2}\right)\left(c-u_{\text {therm }}\right)-g^{\prime}=0,
\end{aligned}
$$

where $u_{\text {therm }}$ is the velocity of the EUC at the thermocline and $\gamma_{1}$, $\gamma_{2}$ are the vorticities in the two layers. This is a quadratic equation in $c$, with the two branches of solutions shown in Figure 5. The upper (lower) branch moves eastward (westward) relative to the thermocline, and extremely short waves on both branches travel near the speed $u_{\text {therm }}$ at the thermocline.

This dispersion relation (1) is quadratic in $c$ and hence trivial to solve explicitly. The $k$-dependence occurs only through the monotone function $k\left(\operatorname{coth} k h_{1}+\operatorname{coth} k h_{2}\right)$, and so solving for $k$ numerically is also straightforward. The dispersion relation for the Constantin-Johnson model is quartic in $c$ with a much more involved dependence on $k$ and, without making any further simplifying assumptions, its resolution is quite delicate.

Despite its relative simplicity, (1) is extremely accurate. In Figure 5, for instance, the error in using (1) rather than the Constantin-Johnson dispersion relation to calculate the wave speed is less than $0.6 \mathrm{~cm} \mathrm{~s}^{-1}$ or about $0.3 \%$ (see Figure 6). In this comparison we have left out waves shorter than $5 \mathrm{~m}$ because our naive evaluation of the Constantin-Johnson formula encounters overflow errors. For longer waves there is no such difficulty, 
and indeed the above error bounds are valid for wavelengths of $100 \mathrm{~km}$ and above.

It is interesting to note that (1) is the Boussinesq limit of the dispersion relation for a two-layer fluid with a rigid lid reported by Compelli and Ivanov (2017). However, we do not assume the free surface is completely flat.

\section{Surface and Thermocline}

While our dispersion relation (1) could have come from a rigidlid model, we are nevertheless able to predict the nonzero, albeit small, displacements of the free surface. Similar formulas exist for $1 \frac{1}{2}$-layer shallow water equations, and are used to estimate the depth of the thermocline from satellite observations of sea level height (Fedorov and Brown, 2009). Our formula, however, contains information about the vertical structure of the EUC and in particular about the presence of critical layers.

Letting $\eta_{1}, \eta_{2}$ be the displacement of the thermocline and the free surface as in Figure 2, our approximation gives

$$
\frac{\eta_{2}}{\eta_{1}}=-\frac{\left(c-u_{\text {therm }}\right)\left(c-u_{\text {surf }}\right) k}{g \sinh k h_{2}} .
$$

While the right-hand side of (2) is small, on the order of $\Delta \rho / \rho$ $=5 \times 10^{-3}$, it can in principle take on both positive and negative values. When it is positive, the crests and troughs of the free surface and thermocline are in phase, and when it is negative, they are $180^{\circ}$ out of phase (see Figure 7). From Figure 8, we see that (2) is almost always negative, but the zoom in Figure 9 shows that it can be positive for wavelengths between $100 \mathrm{~m}$ and $200 \mathrm{~m}$.

As with the dispersion relation, the analogous formula for the Constantin-Johnson-type model is slightly too long to comfortably reproduce here. Like the dispersion relation, it is extremely well approximated by (2), differing by less than $5 \times 10^{-6}$ (see Figure 10).

\section{Critical Layers}

As mentioned in the introduction, critical layers occur when wave speed $c$ coincides with the velocity of the EUC. In our model the EUC has a maximum velocity of $u_{\text {therm }}=1 \mathrm{~m} \mathrm{~s}^{-1}$ and a minimum velocity of $u_{\text {surf }}=-.25 \mathrm{~m} \mathrm{~s}^{-1}$ (see Figure 3 ), and so this is equivalent to $u_{\text {surf }}<c<u_{\text {therm }}$. For the parameter values we have taken, this occurs only for waves shorter than $300 \mathrm{~m}$ (see Figure 9). As usual for an inviscid model, these critical layers expand into the "Kelvin's cat's eye" patterns shown in red in Figure 1.

Looking at (2), we see that critical layers are present only if $\eta_{2} / \eta_{1}>0$, that is, if and only if the crests and troughs of the free surface and the thermocline are in phase. Said another way, the lack of a phase shift between the surface and thermocline is a signature of the presence of critical layers. Because the extremely small free-surface deviations are not visible in Figures 1 or 4, we have illustrated this property schematically in Figure 7. It would be interesting to have a physical explanation of this phenomenon, and to do a more detailed comparison with available data.
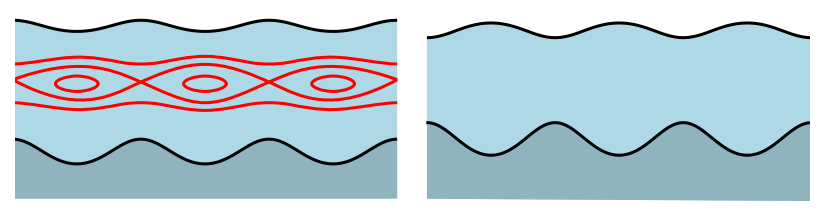

FIGURE 7. (left) The free surface and the thermocline are in phase, so by (2) there is a critical layer, sketched in red. (right) The free surface and the thermocline are out of phase, and so there is no critical layer.

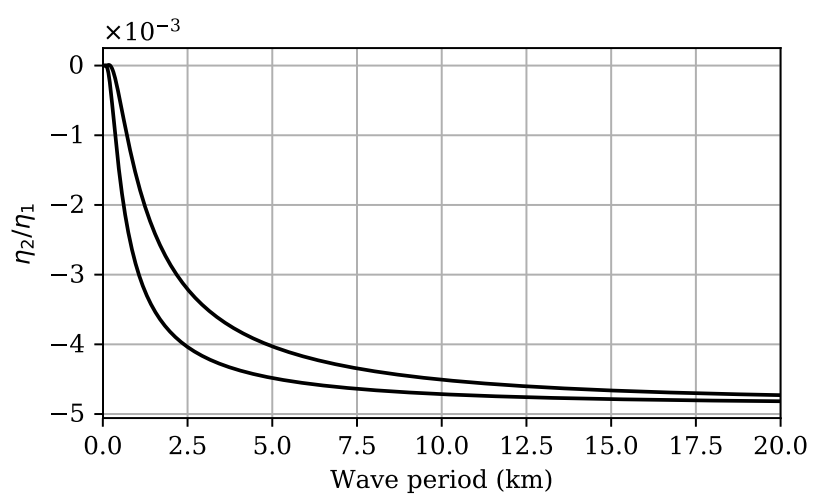

FIGURE 8. The ratio $\eta_{2} / \eta_{1}$ between the amplitude of the surface and the amplitude of the thermocline. As predicted by dimensional analysis, these ratios are on the order of $\Delta \rho / \rho=5 \times 10^{-3}$. Positive values indicate that crests and troughs are in phase, while negative values indicate that they are perfectly out of phase. The upper branch here corresponds to the lower branch in Figure 5.
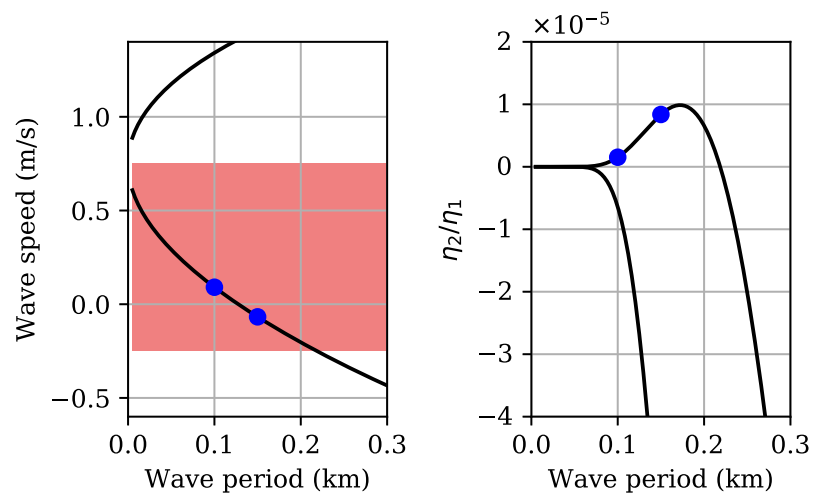

FIGURE 9. Zooms of Figures 5 and 8 for shorter waves. Waves in the red shaded region on the left have critical layers, and the two blue points correspond to the waves shown in Figure 1.

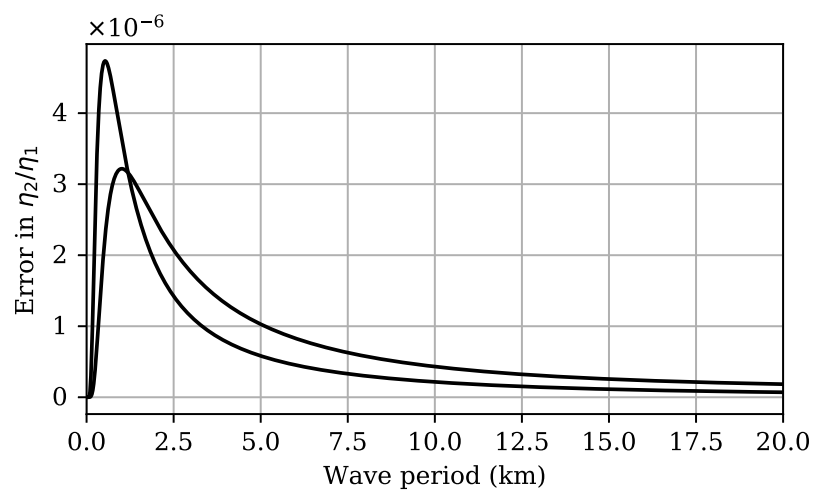

FIGURE 10. Absolute error between the values of $\eta_{2} / \eta_{1}$ in Figure 8 and the corresponding values in the Constantin-Johnsontype model. Note the difference in scales. 


\section{Surface Modes}

One feature of the Constantin-Johnson-type model that our approximation completely misses is the existence of fast waves concentrated near the surface. These come from the other two branches of the full quartic dispersion relation, plotted in Figure 11. As the wavelength increases, the wave speeds continue to increase and approach limits close to the shallow water speed, $\pm \sqrt{g\left(h_{1}+h_{2}\right)} \approx 200 \mathrm{~m} \mathrm{~s}^{-1}$. See Constantin (2011) for a discussion of applications to tsunamis.

These fast waves are filtered out by our model because their wave speeds are much larger than the velocity scale $U=1 \mathrm{~m} \mathrm{~s}^{-1}$ for the EUC. Performing a dimensional analysis with $100 \mathrm{~m} \mathrm{~s}^{-1}$ as the velocity scale, we expect that these waves are only slightly affected by stratification and the EUC. It is therefore reasonable to initially approximate them as gravity waves in a homogeneous fluid otherwise at rest, which have the well-known dispersion relation

$$
c^{2}=g \frac{\tanh \left(\left(h_{1}+h_{2}\right) k\right)}{k} .
$$

Indeed, for wavelengths larger than $200 \mathrm{~m}$, (3) approximates these branches of the full dispersion relation in Figure 11 with an error of less than $0.8 \%$.

\section{The Role of the Coriolis Forces}

While Coriolis forces, and hence Earth's angular velocity, do appear in our model, they are absent from formulas such as (1) and (2). This is not because we have treated the dimensionless parameter

$$
\frac{\Omega L}{U} \approx 7 \times 10^{-2}
$$

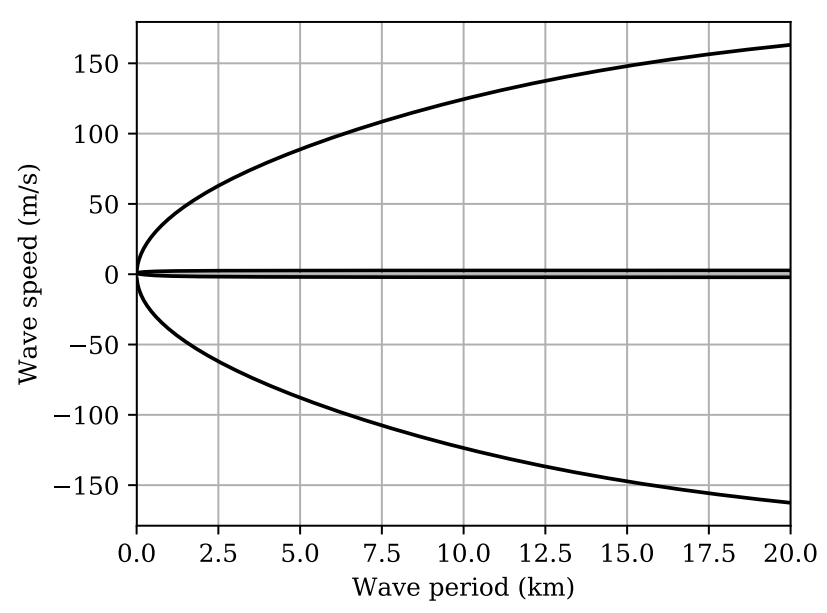

FIGURE 11. All four branches of the Constantin-Johnson dispersion relation. The middle two branches of more slowly traveling waves are approximated to within $0.3 \%$ by (1). For waves longer than $200 \mathrm{~m}$, the remaining two branches are approximated to within $0.8 \%$ by the even simpler dispersion relation (3). as small; indeed we have treated it as an order one quantity. Instead, it is explained by the following observation: For a two-dimensional traveling wave on the equatorial $f$-plane, including Coriolis forces is essentially equivalent to replacing the gravitational constant $g$ by $g-2 \Omega$ c. The more appropriate dimensionless measure of $\Omega$ is therefore

$$
\frac{\Omega c}{g} \sim \frac{\Omega L}{U} \frac{\Delta \rho / \rho}{\mathrm{Ri}} \approx 5 \times 10^{-6} .
$$

At the current level of approximation, this is treated as zero because it contains a factor of $\Delta \rho / \rho$. We emphasize that the above argument does not generalize to time-dependent solutions or indeed to three-dimensional waves of any kind.

\section{CONCLUSION}

We have considered a model in the spirit of Constantin and Johnson (2015) for the interaction of waves and the EUC, together with its Boussinesq approximation. Unlike the various long and short wave limits discussed by Constantin and Johnson (2015), this approximation is extremely accurate for all wavelengths larger than $5 \mathrm{~m}$. Armed with much simpler formulas, we can more easily address important issues such as the validity of rigid-lid approximations, the presence or absence of critical layers, and the influence of Coriolis forces. For simplicity, we have used a piecewise-linear model of the EUC with only two layers, but the method generalizes to any number of layers (at the cost of increasingly complicated formulas).

The main phenomenon that is lost in the approximation is the presence of fast waves concentrated near the surface. On the other hand, these are not particularly influenced by the EUC or by the presence of the thermocline. It is also important to emphasize that, unlike in a rigid-lid model, we do retain information about the small free-surface disturbances associated with large disturbances of the thermocline.

Boussinesq approximations sometimes lead to erroneous conclusions (Long, 1965), and in our case the limit is in some sense singular. It would therefore be worthwhile to justify our formal calculations with a rigorous existence proof. This would necessitate going further into the expansion in $\Delta \rho / \rho$ to include nonlinear terms, and is the subject of ongoing work. @

\section{REFERENCES}

Basu, B. 2018. On a three-dimensional nonlinear model of Pacific equatorial ocean dynamics: Velocities and flow paths. Oceanography 31(3):51-58, https://doi.org/10.5670/oceanog.2018.309.

Compelli, A., and R. Ivanov. 2015. On the dynamics of internal waves interacting with the equatorial undercurrent. Journal of Nonlinear Mathematical Physics 22(4):531-539, https://doi.org/10.1080/14029251.2015.1113052.

Compelli, A., and R. Ivanov. 2017. The dynamics of flat surface internal geophysical waves with currents. Journal of Mathematical Fluid Mechanics 19(2):329-344, https://doi.org/10.1007/s00021-016-0283-4.

Constantin, A. 2011. Nonlinear Water Waves with Applications to Wave-Current Interactions and Tsunamis. CBMS-NSF Regional Conference Series in Applied Mathematics, vol. 81, Society for Industrial and Applied Mathematics (SIAM), Philadelphia, PA, 327 pp, https://doi.org/10.1137/1.9781611971873. 
Constantin, A., R.I. Ivanov, and C.-I. Martin. 2016. Hamiltonian formulation for wave-current interactions in stratified rotational flows. Archives for Rational Mechanics and Analysis 221(3):1,417-1,447, https://doi.org/10.1007/ s00205-016-0990-2.

Constantin, A., and R.S. Johnson. 2015. The dynamics of waves interacting with the Equatorial Undercurrent. Geophysical \& Astrophysical Fluid Dynamics 109:311-358, https://doi.org/10.1080/03091929.2015.1066785.

Constantin, A., and R.S. Johnson. 2016. An exact, steady, purely azimuthal flow as a model for the Antarctic Circumpolar Current. Journal of Physical Oceanography 46:3,585-3,594, https://doi.org/10.1175/JPO-D-16-0121.1.

Craig, W., P. Guyenne, and H. Kalisch. 2005. Hamiltonian long-wave expansions for free surfaces and interfaces. Communications on Pure and Applied Mathematics 58(12):1,587-1,641, https://doi.org/10.1002/cpa.20098.

Fedorov, A.V., and J.N. Brown. 2009. Equatorial waves. Pp. 271-287 in Encyclopedia of Ocean Sciences, 2nd ed. J.H. Steele, ed., Academic Press, https://doi.org/10.1016/B978-012374473-9.00610-X.

Ionescu-Kruse, D., and C.I. Martin. 2017. Periodic equatorial water flows from a Hamiltonian perspective. Journal of Differential Equations 262(8)4,451-4,474, https://doi.org/10.1016/j.jde.2017.01.001.

Johnson, G.C., M.J. McPhaden, and E. Firing. 2001. Equatorial Pacific Ocean horizontal velocity, divergence, and upwelling. Journal of Physical Oceanography 31(3):839-849, https://doi.org/10.1175/1520-0485(2001)031 $<0839$ :EPOHVD>2.0.CO;2.

Long, R.R. 1965. On the Boussinesq approximation and its role in the theory of internal waves. Tellus 17:46-52, https://doi.org/10.3402/tellusa.v17i1.9016.

Maslowe, S.A. 1986. Critical layers in shear flows. Annual Review of Fluid Mechanics 18:405-432, https://doi.org/10.1146/annurev.fl.18.010186.002201. McCreary, J.P. 1985. Modeling equatorial ocean circulation. Annual Review of Fluid Mechanics 17(1):359-409, https://doi.org/10.1146/annurev.fl.17.010185.002043.

McPhaden, M.J., J.A. Proehl, and L.M. Rothstein. 1986. The interaction of equatorial Kelvin waves with realistically sheared zonal currents. Journal of Physical Oceanography 16(9):1,499-1,515, https://doi.org/10.1175/1520-0485(1986)016 $<1499$ :TIOEKW>2.0.CO;2.

\section{ACKNOWLEDGMENTS}

The author thanks the two anonymous referees for their helpful comments.

\section{AUTHOR}

Miles H. Wheeler (miles.wheeler@univie.ac.at) is a researcher at the University of Vienna, Austria.

\section{ARTICLE CITATION}

Wheeler, M.H. 2018. Simplified models for equatorial waves with vertical structure. Oceanography 31(3):36-41, https://doi.org/10.5670/oceanog.2018.307. 